\title{
Neutrino masses, cosmological bound and four zero Yukawa textures
}

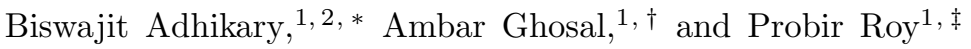 \\ ${ }^{1}$ Saha Institute of Nuclear Physics, 1/AF Bidhannagar, Kolkata 700064, India \\ ${ }^{2}$ Department of Physics, Gurudas College, Narkeldanga, Kolkata 700054, India
}

\begin{abstract}
Four zero neutrino Yukawa textures in a specified weak basis, combined with $\mu \tau$ symmetry and type-I seesaw, yield a highly constrained and predictive scheme. Two alternately viable $3 \times 3$ light neutrino Majorana mass matrices $m_{\nu A} / m_{\nu B}$ result with inverted/normal mass ordering. Neutrino masses, Majorana in character and predicted within definite ranges with laboratory and cosmological inputs, will have their sum probed cosmologically. The rate for $0 \nu \beta \beta$ decay, though generally below the reach of planned experiments, could approach it in some parameter region. Departure from $\mu \tau$ symmetry due to RG evolution from a high scale and consequent CP violation, with a Jarlskog invariant whose magnitude could almost reach $6 \times 10^{-3}$, are explored.
\end{abstract}

PACS numbers: 14.60.Pq; 11.30.Hv; 98.80.Cq

Cosmological observations provide a powerful tool [1] to probe certain properties of elementary particles. Such is a fortiori the case [2] [4] with the sum of the three light neutrino masses $m_{1}, m_{2}, m_{3}$. Its cosmological determination will be of general interest since stable light neutrinos comprise hot dark matter in the Universe. Moreover, it will pin down the absolute scale of the new physics [5] beyond the Standard Model already discovered from neutrino flavor conversion and oscillation experiments. The cosmological upper limit on the said mass sum has recently been claimed, by inclusion of data from large scale redshift survey, to be [6] $0.28 \mathrm{eV}$ within a $95 \%$ c.l. Given the uncertainties [7] due to priors, a value like $0.5 \mathrm{eV}$ is generally accepted 8 now. Further proposed observations 911 aim to bring this bound down by more than half an order magnitude. KATRIN, an experiment on tritium $\beta$-decay in preparation [12], aims to probe the absolute neutrino mass scale down to $0.2 \mathrm{eV}$. Meanwhile, solar as well as atmospheric, reactor and accelerator neutrino and antrineutrino experiments have measured [13] the mass squared differences $\left|\Delta_{32}^{2}\right| \simeq 2.4 \times 10^{-3}$ $\mathrm{eV}^{2}$ and $\Delta_{21}^{2} \simeq 7.6 \times 10^{-5} \mathrm{eV}^{2}, \Delta_{i j}^{2} \equiv m_{i}^{2}-m_{j}^{2}$, leading to a lower bound $\sim 0.05 \mathrm{eV}$ on the mass sum. Within an order of magnitude (from 0.05 to $0.5 \mathrm{eV}$ ) then, the sum of the neutrino masses remains undetermined.

This note is aimed at drawing attention to allowed $\mu \tau$ symmetric four zero Yukawa textures 14 from which, via type-I seesaw [15], the above mass sum as well as individual neutrino masses obtain in terms of either of the mass squared differences. Such four zero Yukawa textures have a two-fold importance. First, four is the maximal number of zeroes phenomenologically allowed [16] in the neutrino Yukawa coupling matrix. Second, they completely fix [16] the high energy CP violating phases needed for leptogenesis in terms of low energy phases observable in the laboratory. Though the framework used here was developed by us earlier [14, we now present several new and interesting results. Apart from the expressions for the individual neutrino masses, we explicitly calculate the two Majorana phases as well as the effective mass $m_{\beta \beta}$ relevant to $0 \nu \beta \beta$ decay and the Jarlskog invariant $J_{\mathrm{CP}}$ for CP violation. We assume only three light neutrinos, identical masses for neutrinos and antineutrinos and the absence of any new nonstandard interaction. The literature today is flush with neutrino mass and mixing models [17. However, the idea of $\mu \tau$ symmetric four zero Yukawa textures provides a novel angle to view the concerned phenomena and probe the mystery of large flavor mixing in the lepton sector. This is especially since a texture postulate is more basic in a coupling matrix appearing in the Lagrangian rather than in the seesaw-derived neutrino mass matrix. Another reason for highlighting this model and these calculations is that the high scale CP violation, needed to describe leptogenesis, is determined [14] here totally by the low energy $\mathrm{CP}$ violation observable in laboratory experiments.

We start with a tabulated summary of the esential features of $\mu \tau$ symmetric four zero Yukawa textures [12]. The new results presented in this communication are explicit expressions for the neutrino masses $m_{1}, m_{2}, m_{3}$ as well as for the neutrino Majorana phases and the derivation of numerical bounds on them. Similar results are also presented on $0 \nu \beta \beta$ decay and $\mathrm{CP}$ violation. In a nutshell, the three neutrino masses in our scheme may be written as

$$
m_{1,2}=\left|\Delta_{21}^{2}\left(\frac{2-X_{3} \mp X}{2 X}\right)\right|^{1 / 2}, m_{3}=\left|\Delta_{21}^{2} / X\right|^{1 / 2} .
$$

(The masses can be rewritten in terms of $\left|\Delta_{32}^{2}\right|, X$ and $X_{3}$.) Here $X$ and $X_{3}$ are dimensionless parameters which are numerically restricted by neutrino oscillation data. With that and the earlier mentioned inputs, the predicted interval for $m_{1}+m_{2}+m_{3}$ (using $3 \sigma$ oscillation data) is $[0.156,0.5] \mathrm{eV} /[0.074,0.132] \mathrm{eV}$ for inverted/normal mass ordering with $\left(\Delta_{32}^{2}<0\right) /\left(\Delta_{32}^{2}>0\right)$.

There appears to be something magical about four zero Yukawa textures. Their phenomenological success vis-a-vis up and down type quark Yukawas is well-documented [18 21. In the lepton sector it is convenient to work 22] in a 


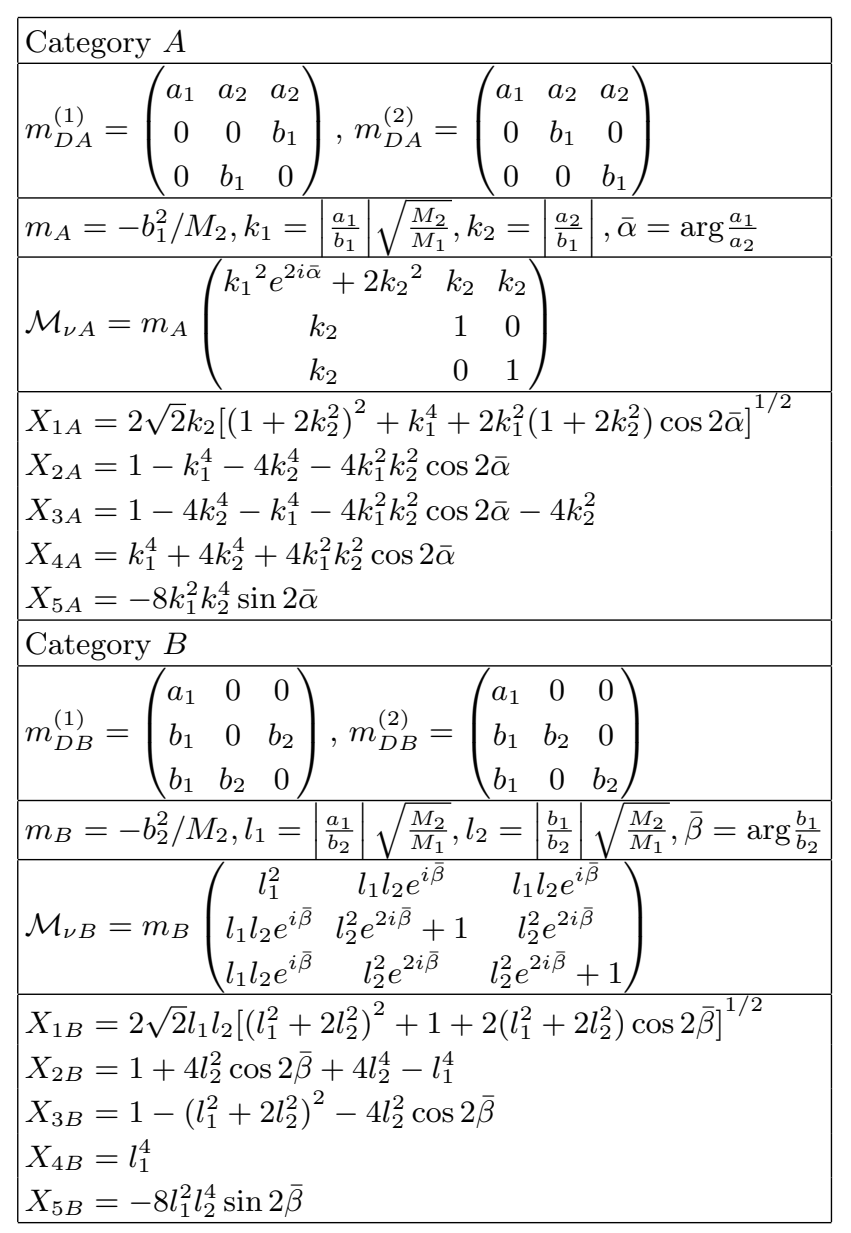

TABLE I: Relevant matrices as well as parameters and their functions in either category

flavor basis with mass diagonal charged leptons $l_{\alpha}(\alpha=e, \mu, \tau)$. Within type-I seesaw, that weak basis gets completely specified by the additional choice [16] of three mass diagonal heavy right chiral neutrinos $N_{i}(i=1,2,3)$ with real masses $M_{i}$. Now four has been demonstrated [16] to be the maximum number of texture zeroes allowed in the neutrino Yukawa coupling matrix $Y_{\nu}$ in flavor space - given the requirement of three lightly massive neutrinos and no unmixed neutrino flavor. High scale CP violation, as required for leptogenesis, then gets completely determined [16] in terms of phases that are in principle measurable in laboratory experiments.

Such a scheme of four zero $Y_{\nu}$ textures is both strongly constrained [23, 24] by neutrino oscillation data and is at the same time quite predictive regarding radiative charged lepton decays. The additional introduction of $\mu \tau$ symmetry [14, motivated by the observed near maximality of $\nu_{\mu}-\nu_{\tau}$ mixing, drastically reduces the number of the allowed textures from seventy two to four. The four allowed textures fall pairwise into two categories $A$ and $B$ with a single light neutrino Majorana mass matrix $\mathcal{M}_{\nu}$ for each category. Apart from one nontrivial phase $\bar{\alpha} / \bar{\beta}$, and an overall mass factor $m_{A} / m_{B}, \mathcal{M}_{\nu A} / \mathcal{M}_{\nu B}$ is characterized by two real parameters $k_{1,2} / l_{1,2}$. The same phase contributes to $0 \nu \beta \beta$ decay, leptogenesis [14, 23, 25] as well as to $\mathrm{CP}$ violation to be studied in long baseline experiments - the latter with the introduction of small departures [14] from $\mu \tau$ symmetry.

We choose to work within the Minimal Supersymmetric Standard Model (MSSM [26]) wherein the neutrino Dirac mass matrix is $m_{D}=Y_{\nu} v_{u} / \sqrt{2}$ with $v_{u} \simeq(246 \mathrm{GeV}) \sin \beta$. We have $\left(Y_{\nu}\right)_{12}=\left(Y_{\nu}\right)_{13},\left(Y_{\nu}\right)_{21}=\left(Y_{\nu}\right)_{31},\left(Y_{\nu}\right)_{23}=\left(Y_{\nu}\right)_{32}$, $\left(Y_{\nu}\right)_{22}=\left(Y_{\nu}\right)_{33}$ and $M_{2}=M_{3}$ from $\mu \tau$ symmetry. The type-I seesaw relation $\mathcal{M}_{\nu}=-m_{D} \operatorname{diag}\left(M_{1}^{-1}, M_{2}^{-1}, M_{3}^{-1}\right) m_{D}^{T}$ then yields a $\mu \tau$ symmetric $\mathcal{M}_{\nu}=\mathcal{M}_{\nu}^{T}$, leading immediately to $\theta_{23}=\pi / 4, \theta_{13}=0$. It further follows that $H \equiv \mathcal{M}_{\nu} \mathcal{M}_{\nu}^{\dagger}$ is also $\mu \tau$ symmetric. Details of these results are available elsewhere [14]. Suffice it to summarize the relevant parts in TABLE I. Here $a_{1,2}, b_{1,2}$ are complex parameters. They appear in $m_{D A} / m_{D B}$ reappearing in $\mathcal{M}_{\nu A} / \mathcal{M}_{\nu B}$ in terms of the real parameters $k_{1,2} / l_{1,2}$ and the phases $\bar{\alpha} / \bar{\beta}\left[27\right.$. Furthermore, $X_{a A} / X_{a B}$, with $a=1$ through 5 are explicitly constructed functions of $\left(k_{1}, k_{2}, \bar{\alpha}\right) /\left(l_{1}, l_{2}, \bar{\beta}\right)$ while $X$ equals $\left(X_{1}^{2}+X_{2}^{2}\right)^{1 / 2}$. Their expressions in terms of the parameters $k_{1}, k_{2}, \bar{\alpha}$ (Category $A$ ) or $l_{1}, l_{2}, \bar{\beta}$ (Category $B$ ) are useful because the $\left\{X_{a}\right\}$ are directly related to 
experimentally measurable quantities such as the mass squared differences, mixing angles, effective majorana mass as measured in $0 \nu \beta \beta$ decay and the CP violating Jarlskog invariant. Specifically, $X$ appears in $\Delta_{21}^{2}$ and $X_{3}-X$ in $\Delta_{32}^{2}$. Computed eigenvalues of $H$, namely the squares of the light neutrino masses, lead to (1). Moreover, we have

$$
\Delta_{21}^{2}=|m|^{2} X, \Delta_{32}^{2}=\frac{|m|^{2}}{2}\left(X_{3}-X\right), \theta_{12}=\frac{1}{2} \tan ^{-1} \frac{X_{1}}{X_{2}} .
$$

In (2) $m$ is the overall (complex) mass factor denoted in Table I by $m_{A} / m_{B}$ for Category $A / B$ and $|m|$ turns out to equal $m_{3}$ in either category.

We use the following [28] $3 \sigma$ intervals for $\tan 2 \theta_{12}, \Delta_{21}^{2}$ and $\Delta_{32}^{2}: 1.83 \leq \tan 2 \theta_{12} \leq 4.90,7.07 \times 10^{-5} \mathrm{eV}^{2} \leq \Delta_{21}^{2} \leq$ $8.34 \times 10^{-5} \mathrm{eV}^{2}$ and $2.13 \times 10^{-3} \mathrm{eV}^{2} \leq \Delta_{32}^{2} \leq 2.88 \times 10^{-3} \mathrm{eV}^{2}$ (normal mass ordering), $-2.79 \times 10^{-3} \mathrm{eV}^{2} \leq \Delta_{32}^{2} \leq$ $-2.02 \times 10^{-3} \mathrm{eV}^{2}$ (inverted mass ordering). These permit 25] only inverted (normal) ordering for Category $A(B)$ and allow only a constrained domain in the $k_{1}-k_{2} / l_{1}-l_{2}$ plane. They further restrict [25] the magnitude of the phases to $89.0^{\circ}<|\bar{\alpha}|<90^{\circ}, 87.0^{\circ}<|\bar{\beta}|<90^{\circ}$, allowing a limited region in the $X_{3}-X$ plane. The consequent correlated values of $m_{i}$ are shown in the top panel plots of FIG.1. The limits on the sum of the neutrino masses, quoted at the begining, follow as a result. For the individual masses $m_{i} /(\mathrm{eV}), i=1,2,3$, we have the respective intervals [0.0452, 0.1682], [0.0457, 0.1684], [0.0077, 0.1623] for Category $A$ and [0.0110, 0.0335], [0.0144, 0.0345], [0.0485, 0.0638] for Category $B$. Given the allowed ranges, it is not possible right now to distinguish between the hierarchical and quasidegenerate possibilities. But a future reduction of the ranges could pin this down.

One can now calculate the neutrino Majorana phases [29] from the explicit diagonalization of $\mathcal{M}_{\nu}$. Let us

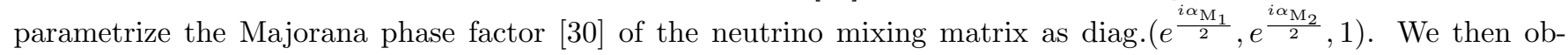
tain

$$
\begin{aligned}
& \cos \left(\alpha_{M_{1}}-\arg . Z\right)=\frac{|Z|^{2} m_{3}^{2}+m_{1}^{2} \sin ^{4} \theta_{12}-m_{2}^{2} \cos ^{4} \theta_{12}}{2 m_{1} m_{3} \sin ^{2} \theta_{12}|Z|} \\
& \cos \left(\alpha_{M_{2}}-\arg . Z\right)=\frac{|Z|^{2} m_{3}^{2}+m_{2}^{2} \cos ^{4} \theta_{12}-m_{1}^{2} \sin ^{4} \theta_{12}}{2 m_{2} m_{3} \cos ^{2} \theta_{12}|Z|}
\end{aligned}
$$

with $Z=\left[\left(\mathcal{M}_{\nu}\right)_{22}+\left(\mathcal{M}_{\nu}\right)_{23}\right]\left[\left(\mathcal{M}_{\nu}\right)_{22}-\left(\mathcal{M}_{\nu}\right)_{23}\right]^{-1}$. Restricting $\alpha_{M_{1}}, \alpha_{M_{2}}$ to the $-\pi$ to $\pi$ interval, utilizing the expressions for $Z, m_{1}, m_{2}, m_{3}$ and $\theta_{12}$ in terms of the basic parameters $\left(k_{1}, k_{2}, \bar{\alpha}\right) /\left(l_{1}, l_{2}, \bar{\beta}\right)$ and utilizing the phenomenologically acceptable ranges of these parameters as given in Ref. [14], we numerically find from eqs.(3) $-98.0^{\circ} \leq \alpha_{M_{1}} \leq 20.0^{\circ}, 9.2^{\circ} \leq \alpha_{M_{2}} \leq 36.4^{\circ}$ for Category $A$ and $-88.6^{\circ} \leq \alpha_{M_{1}} \leq 7.97^{\circ}, 90.7^{\circ} \leq \alpha_{M_{2}} \leq 128.8^{\circ}$ for Category $B$. Allowed values of $\alpha_{M_{2}}$ vs $\alpha_{M_{1}}$ are shown in the middle panels of FIG.1.

The effective mass $m_{\beta \beta}=\left|\left(\mathcal{M}_{\nu}\right)_{11}\right|$, appearing in the transition amplitude for the yet unobserved neutrinoless nuclear double $\beta$-decay, is given in our scheme by

$$
m_{\beta \beta}=\left|\Delta_{21}^{2} X_{4} X^{-1}\right|^{1 / 2}
$$

with $X_{4}$ as appearing in TABLE I. Including uncertainties in the relevant nuclear matrix element, the currently accepted experimental upper bound [31] is $m_{\beta \beta}<0.35 \mathrm{eV}$. In comparison, the predicted $3 \sigma$ ranges for $m_{\beta \beta}$ in our scheme are (cf. FIG.1) $0.038 \leq m_{\beta \beta} / \mathrm{eV} \leq 0.161$ for Category $A$ and $0.003 \leq m_{\beta \beta} / \mathrm{eV} \leq 0.0186$ for Category $B$. Though generally below the planned reach [32, 33] of the forthcoming searches for $0 \nu \beta \beta$ decay, our $m_{\beta \beta}$ could approach it for Category $A$. It could be seriously probed in the subsequent round of experiments. Allowed values of $m_{\beta \beta}$ vs $m_{1}+m_{2}+m_{3}$ are shown in the lowermost panels of FIG.1.

There could be deviation from $\mu \tau$ symmetry and the values $\theta_{23}=\pi / 4, \theta_{13}=0$ might not stand up to more precise forthcoming measurements. Indeed, there is already a hint [13, 28, of a nonzero $\theta_{13}$ from global analyses of neutrino oscillation data. We had earlier proposed [14] a theory of such a departure based on Renormalization Group (RG) evolution from a high energy scale $\Lambda \sim\left(M_{i}\right)_{\text {max }} \sim 10^{12} \mathrm{GeV}$, where $\mu \tau$ symmetry is deemed exact, to a laboratory scale $\lambda \sim 10^{3} \mathrm{GeV}$. The said departure is then caused at the 1-loop level by charged lepton mass terms. Neglecting $m_{\mu, e}^{2}$ in comparison with $m_{\tau}^{2}$, the deviation from $\mu \tau$ symmetry gets characterized by a parameter [34] retained to linear order and given by

$$
\Delta_{\tau} \simeq \frac{m_{\tau}^{2}}{8 \pi^{2} v^{2}}\left(1+\tan ^{2} \beta\right) \ln \frac{\Lambda}{\lambda} \leq 0.05,
$$

the upper bound being reached for [26] $\tan \beta=60$. 

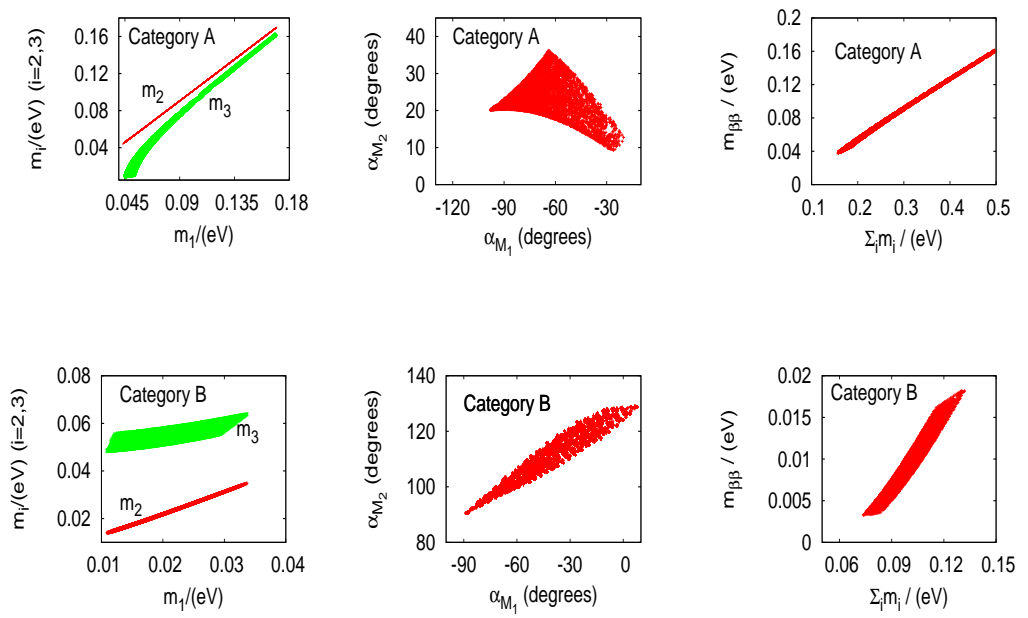

FIG. 1: (Color online) Allowed $m_{2,3}$ vs $m_{1}$ (left), $\alpha_{M_{2}}$ vs $\alpha_{M_{1}}$ (middle) and $m_{\beta \beta}$ vs $\Sigma m_{i}$ (right) for Category $A$ (top) and Category $B$ (bottom).

It now follows that 14

$$
\mathcal{M}_{\nu}^{\lambda} \simeq \operatorname{diag}\left(1,1,1-\Delta_{\tau}\right) \mathcal{M}_{\nu}^{\Lambda} \operatorname{diag}\left(1,1,1-\Delta_{\tau}\right)
$$

where $\mathcal{M}_{\nu}^{\Lambda}$ equals $\mathcal{M}_{\nu}$ of TABLE I and henceforth we drop all superscripts. Detailed expressions to $O\left(\Delta_{\tau}\right)$ for the two neutrino mass squared differences $\Delta_{21}^{2}$ and $\Delta_{32}^{2}$ as well as for the mixing angles $\theta_{i j}$ were given in the Appendix of Ref. [14]. Numerical shifts in neutrino masses and the mass sum are minor, but now $0.0098 \leq m_{\beta \beta} / \mathrm{eV} \leq 0.163$ $\left(0.006 \leq m_{\beta \beta} / \mathrm{eV} \leq 0.0224\right)$ for Category $A(B)$. A nontrivial statement also emerges on the two mixing angles kept free. In Category $A(B)$, we have $36.3^{\circ} \leq \theta_{23}<45^{\circ}\left(45^{\circ}<\theta_{23} \leq 46.8^{\circ}\right)$ and $0<\theta_{13} \leq 2.3^{\circ}\left(0.84^{\circ}\right)$; see the top panels of FIG.2.

Turning to the question of CP violation, which can be observed [35] via a long baseline measurement of the difference in oscillation probabilities $P\left(\nu_{\mu} \rightarrow \nu_{e}\right)-P\left(\overline{\nu_{\mu}} \rightarrow \overline{\nu_{e}}\right)$, our statement on the magnitude of the PMNS phase $\delta$ is $16.0^{\circ} \leq|\delta| \leq 89.50^{\circ}\left(1.50^{\circ} \leq|\delta| \leq 88.05^{\circ}\right)$ for Category $A(B)$. For the basis independent Jarlskog invariant, we have

$$
J_{\mathrm{CP}}=\operatorname{Im} \frac{H_{12} H_{23} H_{31}}{\Delta_{21}^{2} \Delta_{32}^{2} \Delta_{31}^{2}} \simeq \frac{X_{5} \Delta_{\tau}}{X\left(X_{3}^{2}-X^{2}\right)}+O\left(\Delta_{\tau}^{2}\right) .
$$

$X, X_{3}$ in (7) are as before and $X_{5}$ for either category has been given in TABLE I. We find that $0<\left|X_{5}\right| \leq 0.203($ $\left.0<\left|X_{5}\right| \leq 0.0011\right)$, so that $0<\left|J_{\mathrm{CP}}\right| \leq 0.0086\left(0<\left|J_{\mathrm{CP}}\right| \leq 0.0026\right)$ for Category $A(B)$. See the lower panels of FIG. 2 for allowed $|\delta|$ vs $\left|J_{C P}\right|$. The single phase factor $\sin \bar{\alpha} / \sin \bar{\beta}$, appearing $X_{5 A} / X_{5 B}$, is what also shows up [25] in the baryon asymmetry of the Universe originating via high scale leptogenesis. This is a concrete realization of the earlier statement [16] that here CP violation - as required for high scale leptogenesis - is determined by that showing up in laboratory energy neutrino oscillations. The sign of $\delta$ and hence that of $J_{\mathrm{CP}}$ get related via the baryon asymmetry to the mass hierarchy in $N_{i}[23]$.

Let us also address the question of a possible dynamical origin of our scheme. While $\mu \tau$ symmetry is likely to be a discrete subgroup of a broken flavor symmetry group, the real issue is that of the postulated four zero Yukawa textures. In the Frogatt-Nielsen approach [36], one can regard texture zeroes as extremely suppressed entries which can be taken to vanish. On the other hand, a general set of Abelian discrete symmetries to place zeroes in any number of fermionic mass matrix elements was proposed [37, 38, some time ago. A lot of work 39] has in the interim been done with the flavor symmetry groups $A_{4}$ and $S_{3}$, though we are unaware of any model that predicts our $m_{D}$ 's. While there are possibly fruitful directions of interest, a credible understanding of the origin of the present four zero Yukawa textures remains a theoretical challenge.

To sum up, from allowed $\mu \tau$ symmetric four zero Yukawa textures, we have obtained explicit values of the three light neutrino masses in terms of $\sqrt{\Delta_{21}^{2}}$. The predicted magnitude of their sum will soon be tested cosmologically. 

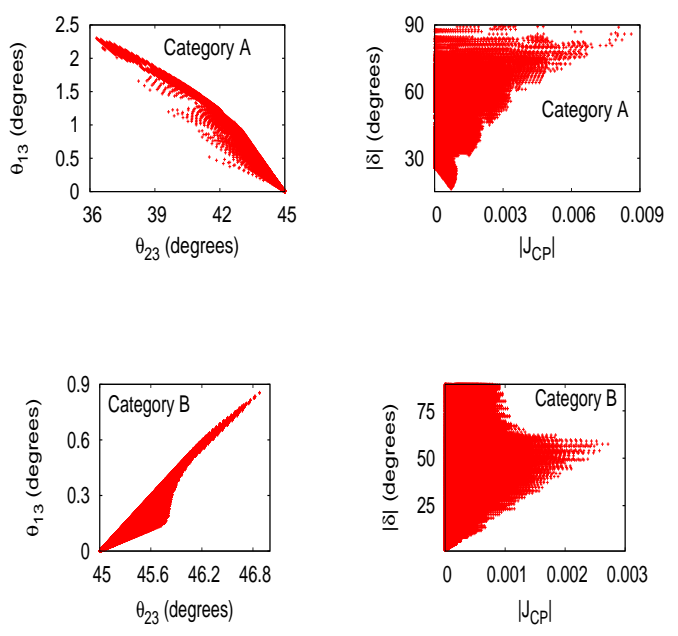

FIG. 2: (Color online) Allowed $\theta_{13}$ vs $\theta_{23}$ (left) and $|\delta|$ vs $\left|J_{C P}\right|$ (right) for Category $A$ (top) and Category $B$ (bottom).

According to our scheme, $0 \nu \beta \beta$ decay may or may not be observed in the near future, but CP violation, to be measured in long baseline experiments at neutrino factories, will tell us how it effected leptogenesis in the early Universe.

Acknowledgements: We thank A. Dighe for discussions on the cosmological bound. P. R. was supported by a DAE Raja Ramanna fellowship.

* Electronic mail: biswajit.adhikary@saha.ac.in

$\dagger$ Electronic mail: ambar.ghosal@saha.ac.in

¥ Electronic mail: probir.roy@saha.ac.in

[1] S. Weinberg, Cosmology, (Oxford University Press, Oxford 2008).

[2] O. Elagory and O. Lahav, New J. Phys. 7, 61 (2005).

[3] J. Lesgourges and S. Pastor, Phys. Rep. 429, 307 (2006).

[4] S. Hannestadt, arXiv:1007.0658 [hep-ph].

[5] See e.g. J. Schechter and J. W. F. Valle, Phys. Rev. D 22, 2227 (1980).

[6] S. A. Thomas, F. B. .Abdalla and O. Lahav, Phys. Rev. Lett. 105, 031301 (2010).

[7] M. C. Gonzalez-Garcia, M. Maltoni and J. Salvado, JHEP 1008, 1117 (2010).

[8] e.g. S. Parke, Unravelling the neutrino mysteries: present and future, Summary talk at NUFACT10, http://www.ino.tifr.res.in/nufact2010/proceedings.php/.

[9] O. Lahav, A. Kiakotou, F. B. Abdalla and C. Blake, arXiv: 0910.4714 [astro-ph].

[10] F. B. Abdalla and S. Rawlings, Mon. Not. R. Astron. Soc. 381, 1313 (2007).

[11] T. Namikawa, S. Saito and A. Taruya, JCAP, 1012, 027 (2010).

[12] http://www-ik.fzk/de/ katrin/index.html.

[13] M. C. Gonzalez-Garcia, M. Maltoni and J. Salvado, JHEP 1004, 056 (2010) and references therein.

[14] B. Adhikary, A. Ghosal and P. Roy, JHEP 0910, 040 (2009) and original references on $\mu \tau$ symmetry therein.

[15] R. N. Mohapatra and P. B. Pal, Massive neutrinos in Physics and Astrophysics (3rd. ed. World Scientific, Singapore 2004) and original references therein.

[16] G. C. Branco, D. Emmanuel-Costa, M. N. Rebelo and P. Roy, Phys. Rev. D 77, 053011 (2008).

[17] For an updated review, see Mu-Chun Chen and K. T. Mahanthappa, arXiv: 1012.1595 [hep-ph].

[18] A review and original references may be found in H. Fritzsch and Z-z. Xing, Prog. Part. Nucl. Phys. 45, 1 (2001).

[19] H. Fritzsch and Z-z. Xing, Phys. Lett. B 555, 63 (2003).

[20] H. Fritzsch, Z-z. Xing and Y. L. Zhou, arXiv:1101.4272 [hep-ph].

[21] K. S. Babu and J. Kubo, Phys. Rev. D 71, 656006 (2005).

[22] G. C. Branco, L. Lavoura and J. P. Silva, CP Violation (Oxford, 1999).

[23] S. Choubey, W. Rodejohann and P. Roy, Nucl. Phys. B 808, 272 (2009); errtm. ibid. 818, 136 (2009).

[24] A. Merle and W. Rodejohann, Phys. Rev. D73, 073012 (2006).

[25] B. Adhikary, A. Ghosal and P. Roy, JCAP, 01, 025 (2011). 
[26] M. Drees, R. M. Godbole and P. Roy, Theory and Phenomenology of Sparticles (World Scientific, Singapore 2004) and references therein.

[27] The phases of $a_{2} / b_{1}$ in Category $A$ and of $a_{1} / b_{2}$ in Category $B$ have been absorbed in the $\nu_{e}$ field.

[28] G. L. Fogli, E. Lisi, A. Marrone, A. Palazzo and A. M. Rotuno, J. Phys. Conf. Sec., 203, 02103 (2010).

[29] B. Adhikary, Phys. Rev. D 74, 033002 (2006).

[30] Particle Physics Booklet, http://pdg.lbl.gov

[31] A. Gando et al., arXiv:1009.4771 [nucl-ex].

[32] J. J. Gomez-Cadenas et al. arXiv:1010:5112 [hep-ex].

[33] S. Schoenert, J. Phys. Conf. Ser. 203012014 (2010).

[34] A. Dighe, S. Goswami and P. Roy, Phys. Rev. D 76096005 (2007).

[35] H. Minakata, Act. Phys. Polon. B 39, 283 (2008).

[36] C. D. Frogatt and H. B. Nielsen, Nucl. Phys. B 147, 2777 (1979).

[37] W. Grimus, A. S. Joshipura, L. Lavoura, M. Tanimoto, Eur. Phys. J. C 36, 227 (2004).

[38] A. Dighe and N. Sahu, arXiv:0812.0695 [hep-ph].

[39] See e.g. F. Gonzalez Canales and A. Mondragon, arXiv:1101.3807 [hep-ph]. 